\title{
Integrated microRNA and proteome analysis reveal a regulatory module in hepatic lipid metabolism disorders in mice with subclinical hypothyroidism
}

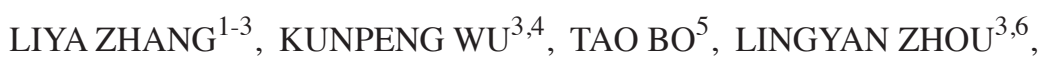 \\ LING GAO ${ }^{2,3,5}$, XIAOMING ZHOU ${ }^{1-3}$ and WENBIN CHEN ${ }^{5}$ \\ ${ }^{1}$ Department of Endocrinology, Shandong Provincial Hospital Affiliated to Shandong University; \\ ${ }^{2}$ Shandong Provincial Key Laboratory of Endocrinology and Lipid Metabolism; ${ }^{3}$ Institute of Endocrinology \\ and Metabolism, Shandong Academy of Clinical Medicine, Jinan, Shandong 250021; ${ }^{4}$ Department of Hematology, \\ Huashan Hospital, Fudan University, Shanghai 200040; ${ }^{5}$ Scientific Center, Shandong Provincial Hospital \\ Affiliated to Shandong University, Jinan, Shandong 250021; ${ }^{6}$ Department of Endocrinology and \\ Metabolism, The Second Hospital of Shandong University, Jinan, Shandong 250033, P.R. China
}

Received November 30, 2018; Accepted November 11, 2019

DOI: $10.3892 / \mathrm{etm} .2019 .8281$

\begin{abstract}
Subclinical hypothyroidism ( $\mathrm{SCH}$ ) is becoming a global health problem due to its increasing prevalence and potential adverse effects, including cardiovascular diseases and nonalcoholic fatty liver disease (NAFLD). However, the association between $\mathrm{SCH}$ and NAFLD remains controversial. MicroRNAs (miRNAs/miRs) have been reported to be implicated in lipid metabolism disorders; however, how miRNAs regulate hepatic lipid metabolism in $\mathrm{SCH}$ mice remains unknown. The present study investigated miRNA alterations and proteome profiles in an SCH mouse model, which was generated by methimazole administration in mice for 16 weeks. Next, the profiles of 17 miRNAs that are critical to hepatic lipid metabolism and the proteome were investigated using reverse transcription-quantitative polymerase chain reaction and iTRAQ labeling in the liver specimens of $\mathrm{SCH}$ $(n=9)$ and control $(n=7)$ mice. Putative target prediction of miRNAs was also conducted using TargetScan and miRanda. Compared with the control mice, SCH mice had 8 miRNAs and 36 proteins with significantly different expression in the liver tissues. Furthermore, a regulatory module containing
\end{abstract}

Correspondence to: $\mathrm{Dr}$ Xiaoming Zhou, Department of Endocrinology, Shandong Provincial Hospital Affiliated to Shandong University, 324 Jing 5 Road, Jinan, Shandong 250021, P.R. China

E-mail: sdslyy@yeah.net

Dr Wenbin Chen, Scientific Center, Shandong Provincial Hospital Affiliated to Shandong University, 324 Jing 5 Road, Jinan, Shandong 250021, P.R. China

E-mail: kuerten1108@163.com

Key words: subclinical hypothyroidism, thyroid stimulating hormone, microRNA, proteome, hepatic lipid metabolism disorder
3 miRNAs (miR-34a-5p, miR-24-3p and miR-130a-3p) and 4 proteins (thioredoxin, selenium-binding protein 2 , elongation factor $1 \beta$ and prosaposin) was identified. Overall, integrated analysis of miRNAs and the proteome highlighted a regulatory module between miRNAs and proteins, which, to a certain extent, may contribute to a better understanding of hepatic lipid metabolism disorders in $\mathrm{SCH}$ mice.

\section{Introduction}

Subclinical hypothyroidism ( $\mathrm{SCH}$ ) is closely associated with disturbances in lipid metabolism, and is characterized by serum thyroid-stimulating hormone (TSH) levels that are above the reference range, while the serum total or free thyroid hormone levels remain within the reference range (1). The prevalence of $\mathrm{SCH}$ ranges between 4 and $20 \%$ of the population in different regions of the world (2). In recent years, a growing body of evidence has indicated that $\mathrm{SCH}$ is an independent risk factor for lipid metabolic disorders, such as cardiovascular diseases and nonalcoholic fatty liver disease (NAFLD) (3). Although there have been studies on the $\mathrm{SCH}$ molecular mechanism, mainly focusing on ligand-receptor interactions and the biological effects at the cellular or molecular levels (4), the underlying mechanism of this condition currently remains unclear.

MicroRNAs (miRNAs/miRs) are small non-coding RNAs with a length of $\sim 18-23$ nucleotides, which interact with mRNAs upon specific base-pairing in the 3'-untranslated region to repress the mRNA expression via translational inhibition or mRNA degradation. miRNAs repress multiple target genes in linear whole pathways or network nodes, thereby simultaneously exerting a larger cumulative effect (5). These small molecules are the focus of basic research on regulating biological processes, as well as of applied research for their potential application as biomarkers and therapeutic agents (6). Recently, accumulating evidence has supported the importance of hepatic miRNAs in the physiological process 
of hepatic lipid metabolism and a wide spectrum of diseases, including viral hepatitis, cirrhosis, hepatoma and NAFLD (7). However, little is known on the role of miRNAs in hepatic lipid metabolic disorders associated with SCH.

Proteomics analysis has been widely used to identify and quantify proteins associated with biological functions that are regulated by miRNAs (8). miRNA target regulatory modules have previously been identified and studied in liver fibrosis (9). However, to the best of our knowledge, no previous study has investigated the regulatory modules in $\mathrm{SCH}$. In the present study, miRNAs alterations and proteome profiles in $\mathrm{SCH}$ were compared and integrated. in $\mathrm{SCH}$ mice. The integration was achieved by targeting predictions at the sequence level.

\section{Materials and methods}

Research animals. Male C57BL/6 mice (age, 7 weeks, weight, 20-21 g) were purchased from Vital River Corporation (Beijing, China) and housed in designated specific-pathogen-free cages on a $12 \mathrm{~h}$ light-dark cycle at $23^{\circ} \mathrm{C}$ and $60 \%$ humidity. Mice were allowed free access to an irradiated chow and sterilized water. After acclimatization for 1 week, the mice were randomly divided into two groups, including the $\mathrm{SCH}$ model $(n=9)$ and control (CON, n=7) groups. The $\mathrm{SCH}$ and $\mathrm{CON}$ groups were given methimazole (MMI; $0.08 \mathrm{mg} / \mathrm{kg}$ body weight per day) or an equal volume of vehicle, respectively, in their drinking water for 16 weeks. Finally, the mice were euthanized using pentobarbital sodium (concentration=20 mg/ml; dose $=120 \mathrm{mg} / \mathrm{kg}$ body weight) through the intraperitoneal route. Following cervical dislocation to ensure death, $600 \mu \mathrm{l}$ blood samples and the liver tissues of mice were collected. All animal experiments were performed according to the relevant guidelines and institutional policies (10). The animal protocol was approved by the Shandong Provincial Hospital Animal Care and Use Committee (no. 2015-003; Jinan, China).

Serum TSH, free thyroxine (FT4), lipid profile and liver function assay. Serum TSH level was determined using a mouse ELISA kit (MyBioSource), following the product manual. Serum FT4 concentrations were determined using specific radioimmunoassay kits (Jiuding Diagnostic). Serum triglyceride (TG), total cholesterol (TC), high-density lipoprotein cholesterol (HDL-C), low-density lipoprotein cholesterol (LDL-C), alanine transaminase (ALT) and aspartate aminotransferase (AST) levels were analyzed using an Olympus AU5400 automatic biochemical analyzer (Olympus Corporation) at Shandong Provincial Hospital.

Hepatic TG and TC assays. The hepatic TG and TC content was analyzed using a TG assay kit (E1013; Applygen Technologies, Inc.) and a TC assay kit (E1015; Applygen Technologies, Inc.), respectively. Briefly, approximately $50 \mathrm{mg}$ of liver samples were cut, homogenized using a manual homogenizer and lysed in TG kit or TC kit lysis buffer for $10 \mathrm{~min}$ at room temperature. After centrifugation at 2,000 $\mathrm{x} g$ for $5 \mathrm{~min}$ at room temperature, the supernatants were divided into two parts, one for TG or TC measurements and the other for protein concentration assay. A bicinchoninic acid (BCA) protein assay was performed using the BCA protein assay kit (Pierce; Thermo Fisher Scientific, Inc.) to determine protein concentration. The final TG or TC levels were standardized by protein concentration and were expressed as $\mu \mathrm{mol}$ per gram of protein.

Oil red $O$ staining. To determine the levels of hepatic lipids, frozen liver sections $(8 \mu \mathrm{m})$ were mounted on slides and air dried for $10 \mathrm{~min}$ at room temperature, then sections were fixed with $10 \%$ buffered formalin for $30 \mathrm{~min}$ at room temperature. After washing with PBS, sections were stained with Oil red $\mathrm{O}$ for $10 \mathrm{~min}$ at room temperature, and then counterstained with hematoxylin for $20 \mathrm{sec}$. Photomicrographs were captured using an imaging system under a microscope (Axiovert 100M; Zeiss AG).

miRNA extraction, reverse transcription (RT) into cDNA and quantitative polymerase chain reaction ( $q P C R)$ for miRNA. Liver miRNAs were extracted from the tissues using the MiRcute miRNA isolation kit (Tiangen Biotech Co., Ltd.), according to the manufacturer's protocol. Next, the concentration of RNA was quantified using a NanoDrop 1000 system (NanoDrop Technologies; Thermo Fisher Scientific, Inc.). For RT into cDNA, the RNA was poly(A)-tailed and reverse transcribed into cDNA using random primers and the miRcute miRNA First-Strand cDNA Synthesis Kit (Tiangen Biotech Co., Ltd.), following which qPCR was performed with the miRcute miRNA qPCR Detection kit (Tiangen Biotech Co., Ltd.) following the manufacturer's protocol with the forward primers presented in Table I. The universal reverse primers were synthesized and provided by Tiangen Biotech Co., Ltd. The PCR thermocycling conditions were as following: Denaturation at $95^{\circ} \mathrm{C}$ for $15 \mathrm{~min}$, followed by 45 cycles of $94^{\circ} \mathrm{C}$ for $20 \mathrm{sec}$ and $60^{\circ} \mathrm{C}$ for $34 \mathrm{sec}$. The cycle quantification $(\mathrm{Cq})$ value was calculated using the second derivative maximum method (11). U6 small nuclear RNA was used as the internal control. The relative expression of each miRNA following normalization was determined as follows: $\mathrm{Cq}$ (U6) $-\mathrm{Cq}$ (miRNA).

Protein preparation and liquid chromatography-tandem mass spectrometry (LC-MS/MS) analysis. Hepatic protein was extracted as previously described (12). Next, protein concentration was determined using the BCA Assay kit (Thermo Fisher Scientific, Inc.). The protein samples from three mice were pooled with the ratio 1:1:1 as a biological sample to avoid erroneous conclusions due to individual variations. Each pool was analyzed in duplicate. A total of $200 \mu \mathrm{g}$ protein from each pool was reduced, alkylated and digested with trypsin. Subsequently, the dried peptides were labeled following the manufacturer's recommendations of the isobaric tags for relative and absolute quantitation (iTRAQ) 4-plex kits (SCIEX, Framingham, MA, USA) with iTRAQ tags, as follows: iTRAQ 114 for CON, iTRAQ 115 for SCH, iTRAQ 116 for CON replicate and ITRAQ 117 for SCH replicate. The eluted peptides were analyzed by LC-MS/MS, according to a previously described protocol (13).

Functional enrichment analysis. For functional analysis of the altered proteins, the proteins were imported into the Database for Annotation, Visualization and Integrated Discovery (DAVID version 6.7; http://david.abcc.ncifcrf.gov/) which involves an integrated biological knowledge base and 
Table I. Forward primer sequences used in reverse transcription-quantitative polymerase chain reaction.

\begin{tabular}{lll}
\hline Name & \multicolumn{1}{c}{ Sequence $\left(5^{\prime}-3 '\right)$} & GenBank no. \\
\hline U6 & CTCGCTTCGGCAGCACA & NR002082.1 $\left({ }^{\circ} \mathrm{C}\right)$ \\
mmu-miR-10b-5p & TACCCTGTAGAACCGAATTTGG & 58 \\
mmu-miR-24-3p & TGGCTCAGTTCAGCAGGAACAG & LM378853.1 \\
mmu-miR-29a-3p & TAGCACCATCTGAAATCGGTTA & LM378864.1 \\
mmu-miR-30b-5p & TGTAAACATCCTACACTCAGCT & LM379104.1 \\
mmu-miR-30c-5p & TGTAAACATCCTACACTCTCAGC & LM378817.1 \\
mmu-miR-34a-5p & TGGCAGTGTCTTAGCTGGTTGT & LM379083.1 \\
mmu-miR-125b-5p & TCCCTGAGACCCTAACTTGTGA & LM379111.1 \\
mmu-miR-130a-3p & CAGTGCAATGTTAAAAGGGCAT & LM378823.1 \\
mmu-miR-148a-3p & TCAGTGCACTACAGAACTTTGT & LM378828.1 \\
mmu-miR-155 & TTAATGCTAATTGTGATAGGGGT & LM379085.1 \\
mmu-miR-199a-5p & CCCAGTGTTCAGACTACCTGTTC & LM608259.1 \\
mmu-miR-206 & TGGAATGTAAGGAAGTGTGTGG & LM378873.1 \\
mmu-miR-210-3p & CTGTGCGTGTGACAGCGGCTGA & LM379180.1 \\
mmu-miR-291b-3p & AAAGTGCATCCATTTTGTTTGT & LM379180.1 \\
mmu-miR-370-3p & GCCTGCTGGGGTGGAACCTGGT & LM379852.1 \\
mmu-miR-467b-5p & GTAAGTGCCTGCATGTATATG & LM379368.1 \\
mmu-miR-486a-5p & TCCTGTACTGAGCTGCCCCGAG & LM380560.1 \\
\hline
\end{tabular}

miR, microRNA; Tm, melting temperature.

analytical tools designed to systematically extract biological meaning from large gene/protein lists in order to perform a Gene Ontology (GO) functional enrichment analysis and a Kyoto Encyclopedia of Genes and Genomes (KEGG) pathway enrichment analysis. Path mining tools such as gene function classification, functional annotation table or clustering were used for analysis. Furthermore, TargetScan (Release 7.2; http://www.targetscan.org/vert_72/) and miRanda (August 2010 release; http://www.microrna.org/microrna/ microrna/home.do) database analyses were employed to identify putative targets of miRNAs among the differentially expressed proteins (14).

Statistical analysis. The data were analyzed using SPSS software (version 23.0; IBM Corp.) and are expressed as the mean \pm standard deviation. Differences between two groups were compared using an unpaired Student's t-test. Cluster version 3.0 and Java TreeView version 1.60 (Stanford University) were used to perform agglomerative hierarchical cluster analysis. All of the calculated P-values were two-sided, and $\mathrm{P} \leq 0.05$ was considered to indicate a statistically significant difference.

\section{Results}

Generation of an SCH mouse model and determination of lipid parameters. An SCH mouse model was established by MMI administration for 16 weeks. The SCH mice exhibited normal serum FT4 levels (Fig. 1A) and higher TSH levels (Fig. 1B) as compared with those in CON mice. The serum ALT (Fig. 1C) and AST (Fig. 1D) levels in SCH mice were similar to those exhibited by the $\mathrm{CON}$ group. In addition, serum TG (Fig. 1E), TC (Fig. 1F) and LDL-C (Fig. 1G) levels in $\mathrm{SCH}$ mice were all significantly higher when compared with those in CON mice, whereas there was no marked difference in serum HDL-C levels (Fig. 1H). Oil red O staining of liver tissues indicated greater lipid droplet accumulation in the livers of SCH mice in comparison with the $\mathrm{CON}$ mice (Fig. 1I). Furthermore, the hepatic TG (Fig. 1J) and TC (Fig. 1K) content were markedly increased in the $\mathrm{SCH}$ mice.

Differentially expressed miRNAs in the livers of $\mathrm{SCH}$ mice. 17 candidate miRNAs involved in hepatic lipid metabolic were selected based on previous studies (15-32) and non-coding RNA sequencing (data not shown). The present study then screened these miRNAs by RT-qPCR. Among these 17 miRNAs, 8 miRNAs were found to be significantly dysregulated in SCH mice, including miR-10b-5p, miR-24-3p, miR-29a-3p, miR-30b-5p, miR-34a-5p, miR-125b-5p, miR-130a-5p and miR-199a-5p (Fig. 2A; P<0.05). The expression of the remaining 9 miRNAs was not markedly different between the $\mathrm{SCH}$ and $\mathrm{CON}$ mice (Fig. 2B). These results suggested that the 8 dysregulated miRNAs may be involved in the development of hepatic lipid metabolic disorders in $\mathrm{SCH}$.

Functional analysis of proteins identified by proteomics. The present study performed iTRAQ-based proteomic analysis in the livers of SCH mice. A fold-change $>1.8$ or $<0.56$ was deemed significantly upregulated or downregulated, respectively. A total of 1,969 proteins were quantified, of which 36 were found to be differentially expressed in the livers of $\mathrm{SCH}$ mice, including 22 upregulated and 14 downregulated proteins, as compared with their expression in control tissues. Next, all 

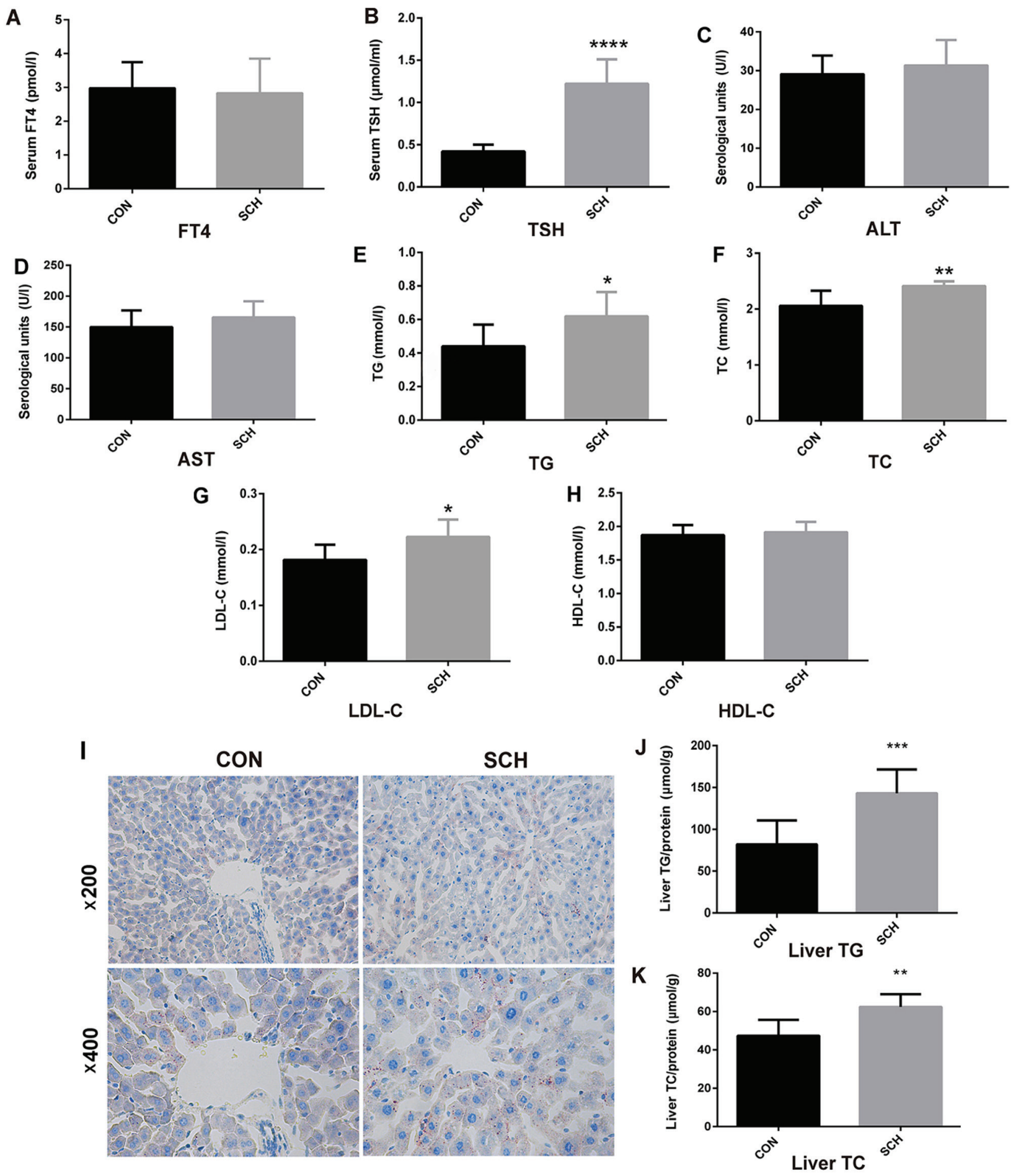

Figure 1. Generation of SCH mouse model and determination of lipid parameters. C57BL $/ 6$ mice were administered methimazole $(0.08 \mathrm{mg} / \mathrm{kg} \mathrm{BW}$ per day) in the SCH group ( $\mathrm{n}=9$ ) or an equal volume of vehicle in the CON group (n=7) for 16 weeks. (A) FT4, (B) TSH, (C) ALT, (D) AST, (E) TG, (F) TC, (G) LDL-C and (H) HDL-C levels in the serum of mice were detected. (I) Oil red O staining of liver tissues (magnification, $\mathrm{x} 200$ and $\mathrm{x} 400$ ). (J) TG and (K) TC content in liver tissues of mice. The data are presented as the mean \pm standard deviation. The TG and TC contents were standardized to the corresponding total protein content in the liver. ${ }^{*} \mathrm{P}<0.05,{ }^{* *} \mathrm{P}<0.01,{ }^{* * *} \mathrm{P}<0.001$ and ${ }^{* * * *} \mathrm{P}<0.0001$ vs. CON group. $\mathrm{SCH}$, subclinical hypothyroidism; CON, control; FT4, free thyroxine; TSH, thyroid-stimulating hormone; ALT, alanine transaminase; AST, aspartate aminotransferase; TG, triglyceride; TC, total cholesterol; LDL-C, low density lipoprotein cholesterol; HDL-C, high density lipoprotein cholesterol.

the differentially expressed proteins were further categorized by DAVID analysis.

By GO functional classification, the identified proteins were clustered into three groups, including proteins involved in biological processes, cell components and molecular functions. In the biological process cluster, the majority of proteins were assigned to 'metabolic process' (38\%) and 'cellular process' (28\%; Fig. 3A). The top two enriched terms in the cellular component cluster included 'cell part' (44\%) and 'organelle' (20\%; Fig. 3B). In the molecular function cluster, a 

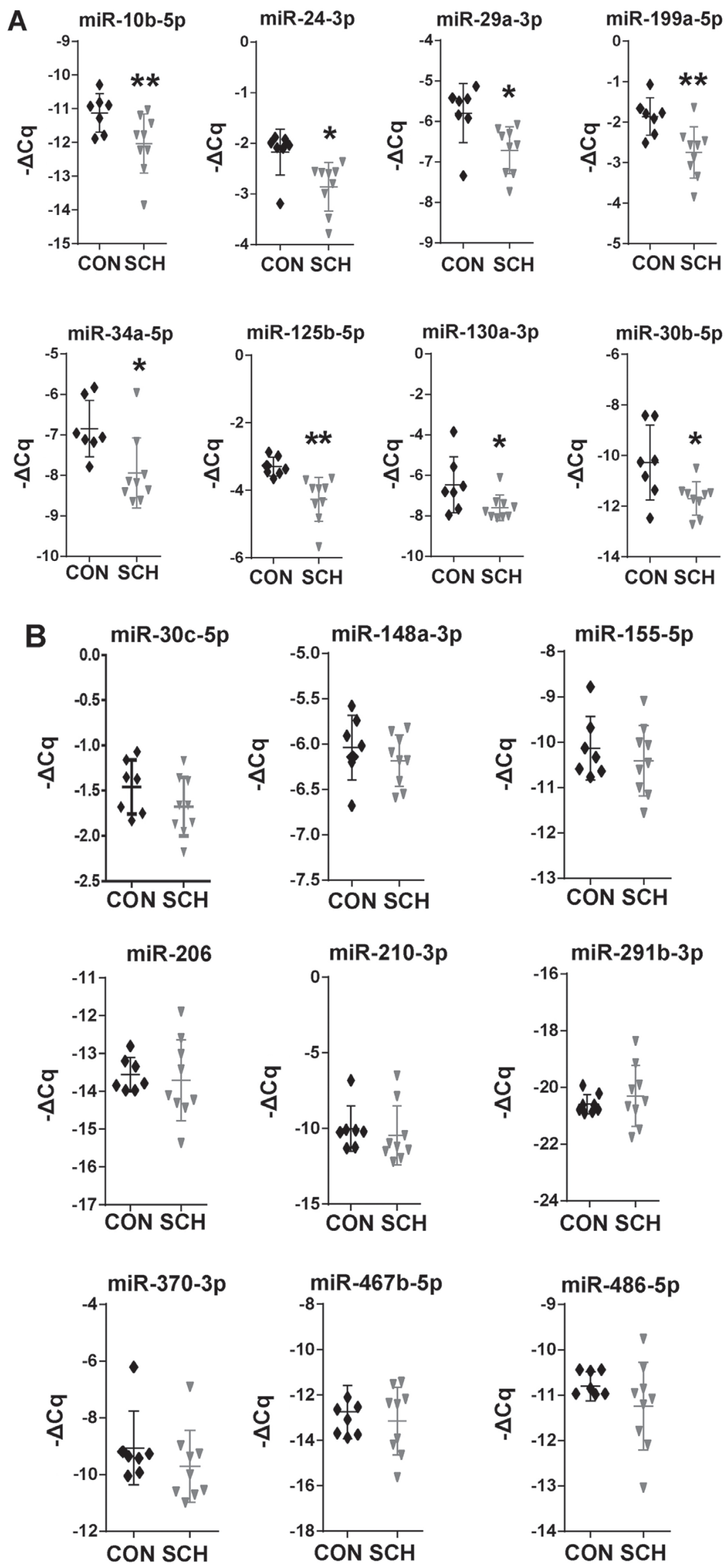

Figure 2. Differentially expressed miRNAs in the livers of SCH mice. (A) A total of 8 miRNAs were downregulated and (B) A total of 9 miRNAs were not markedly changed in the livers of SCH mice. U6 snRNA was used as the internal control. The horizontal lines represent the mean. Statistical significance was assessed using Student's t-test. The data are presented as the mean \pm standard deviation. ${ }^{*} \mathrm{P}<0.05$ and ${ }^{* *} \mathrm{P}<0.01$ vs. CON group ( $=7$ ). miRNA, microRNA; $\mathrm{SCH}$, subclinical hypothyroidism; CON, control. 
A Biological process

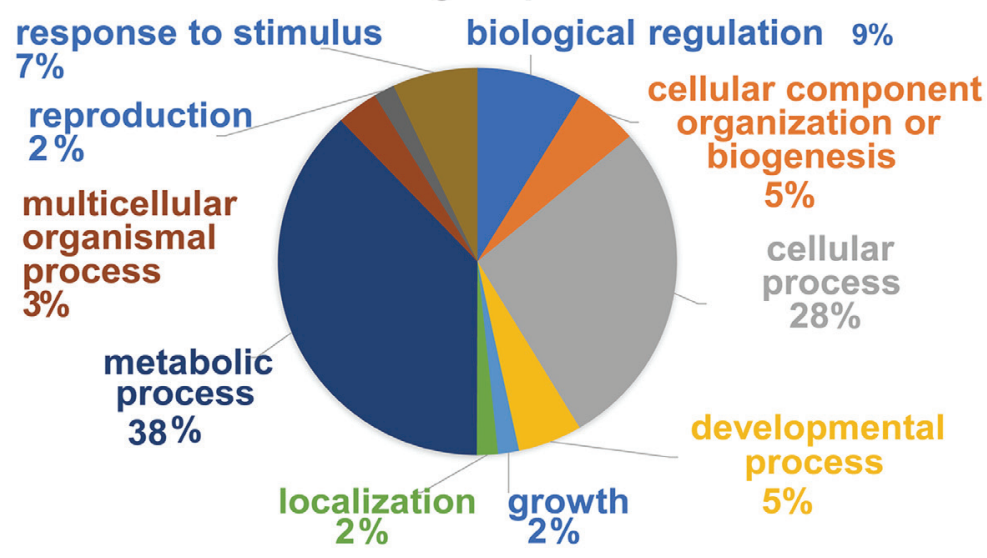

B

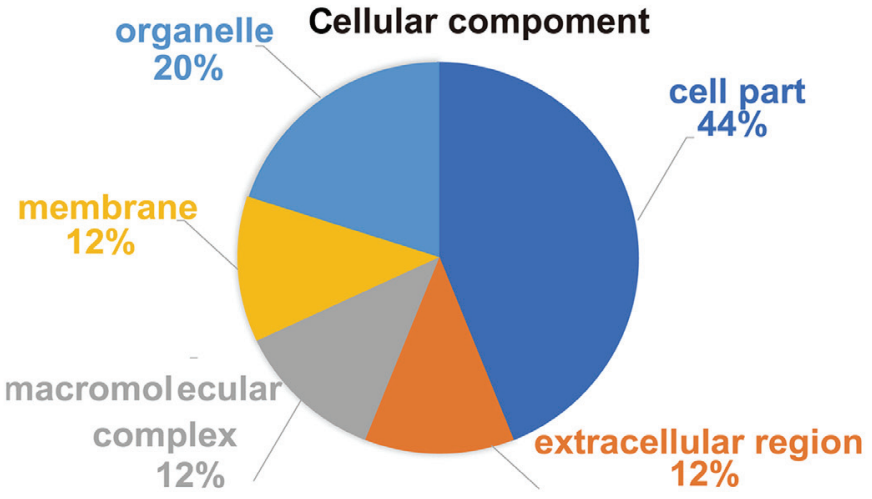

C

\section{Molecular function}
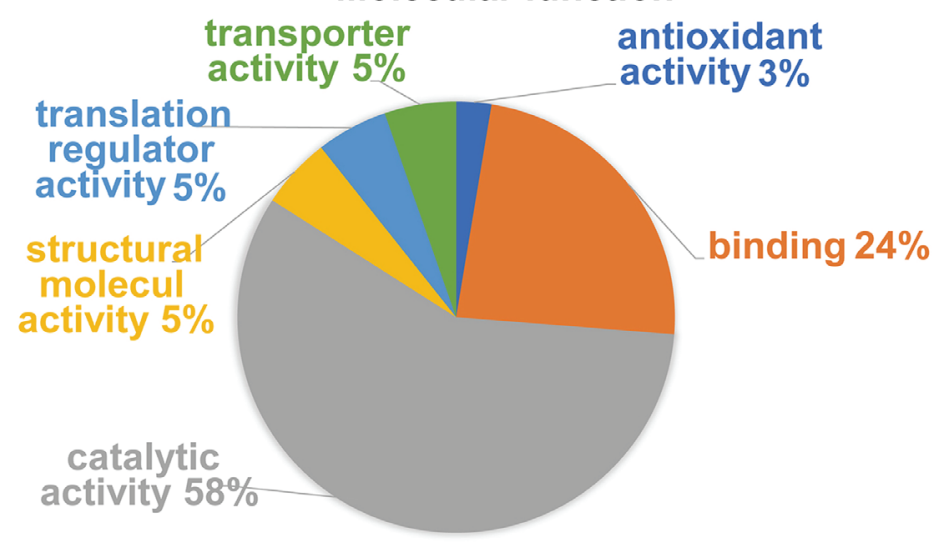

D

KEGG pathway

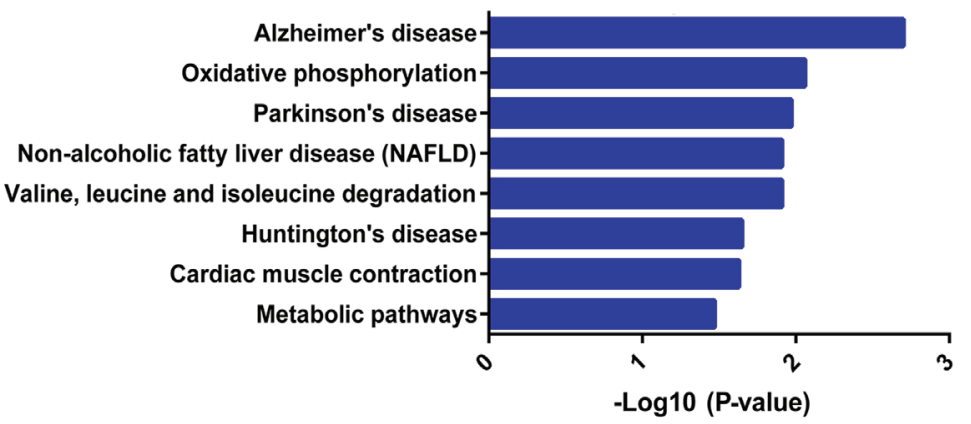

Figure 3. Functional analysis of proteins identified by proteomics. Percentages of proteins enriched in different (A) biological processes, (B) cellular components and (C) molecular functions, according to Gene Ontology enrichment analysis. (D) Results of KEGG pathway analysis. The vertical axis denotes the KEGG pathway categories, and the horizontal axis denotes the negative logarithmic P-value (- $\log _{10} \mathrm{P}$-value), indicating the statistical significance of the pathways based on DAVID analysis. KEGG, Kyoto Encyclopedia of Genes and Genomes. 
A

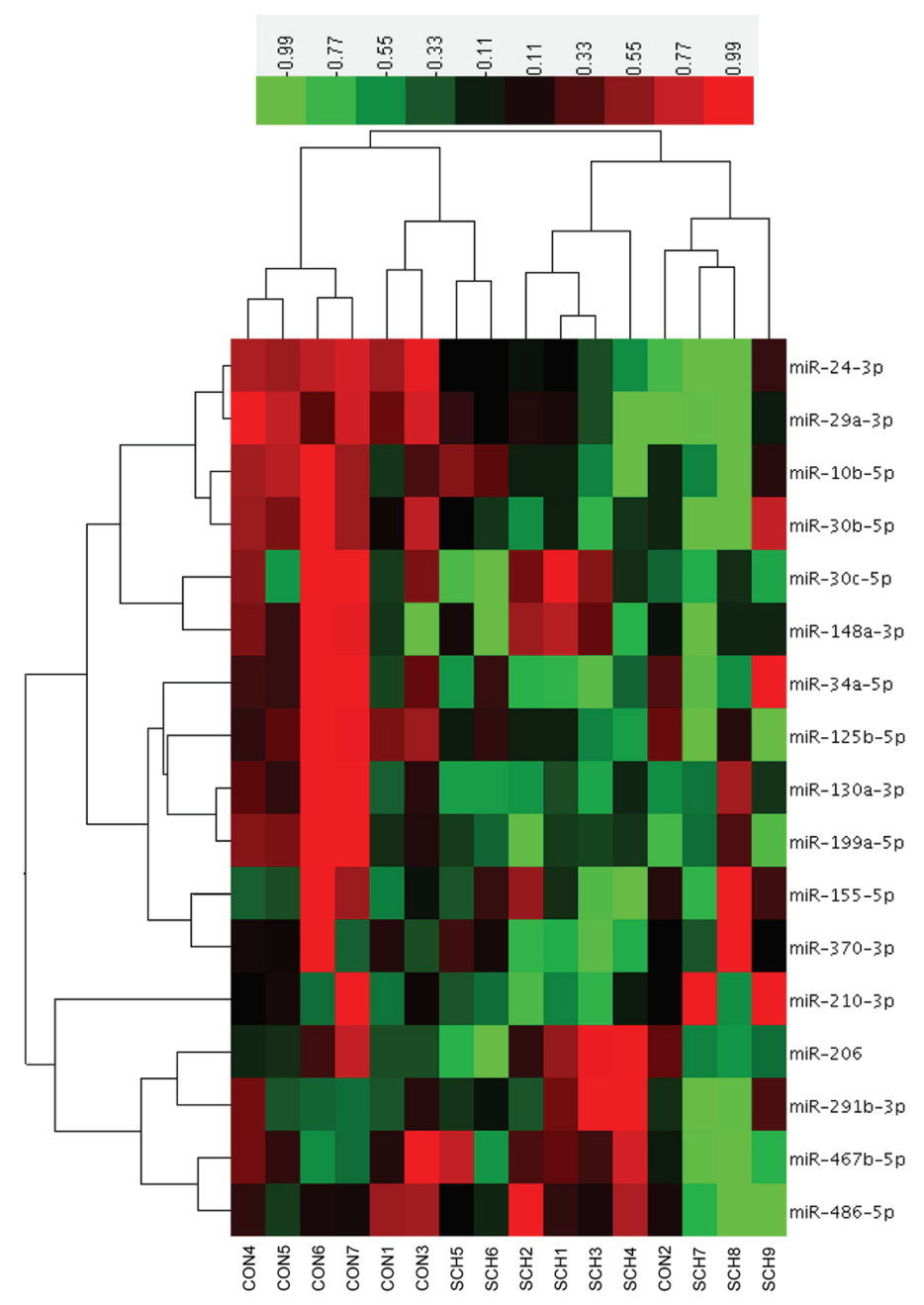

B

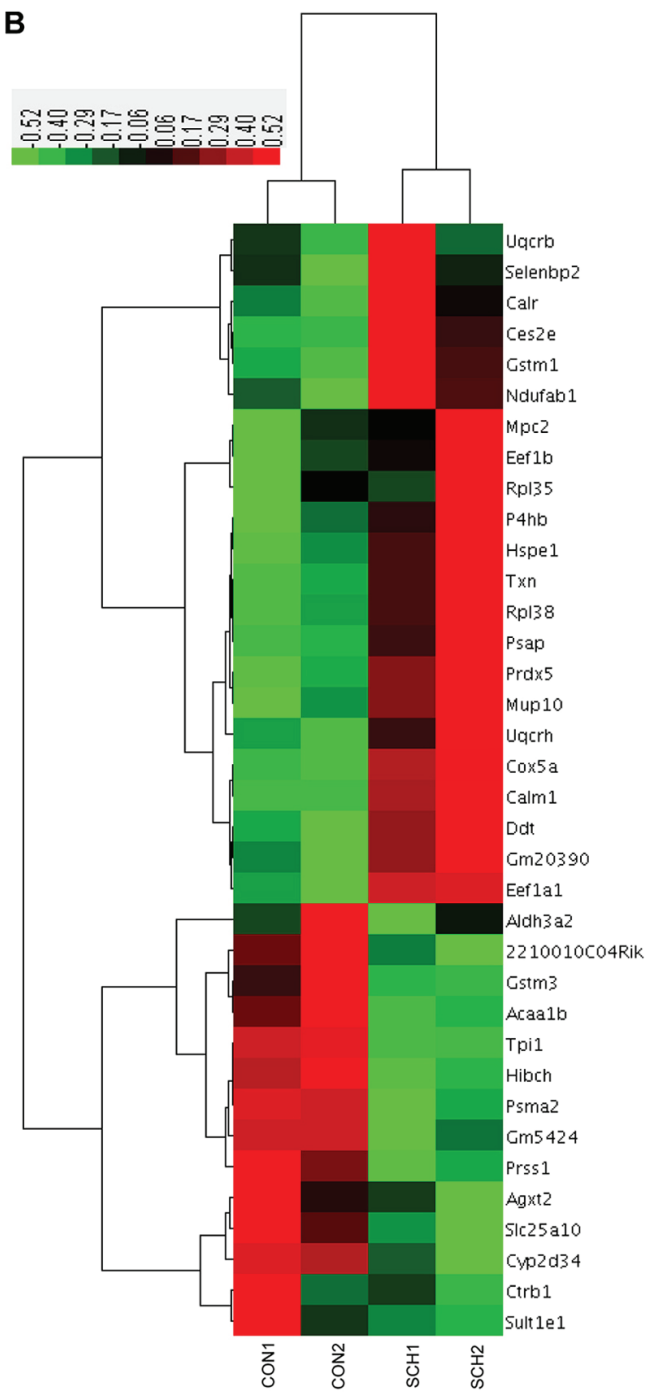

Figure 4. Hierarchical clustering based on the 17 candidate miRNAs and 36 proteins investigated in the study. (A) Hierarchical clustering of the 17 candidate miRNAs. (B) Hierarchical clustering of the differential proteins. Colored areas indicate the relative expression of each sample relative to the mean expression. Red, black and green shading indicates relatively high, unchanged and low expression, respectively. The bar colors represent the scale. miRNA, microRNA; $\mathrm{SCH}$, subclinical hypothyroidism; $\mathrm{CON}$, control.

great number of proteins were assigned to 'catalytic activity' (58\%) and 'binding' (24\%; Fig. 3C). Furthermore, according to KEGG pathway analysis, the majority of the identified proteins were involved in KEGG pathways such as 'Alzheimer's disease', 'oxidative phosphorylation', 'Parkinson's disease' and 'NAFLD' (Fig. 3D). Taken together, the results suggested that these differentially expressed proteins may be important effectors associated with hepatic lipid metabolic disorders in $\mathrm{SCH}$.

Hierarchical clustering based on the 17 candidate miRNAs and 36 proteins. Hierarchical clustering was performed based on the 17 candidate hepatic miRNAs (Fig. 4A). Of the $7 \mathrm{CON}$ mice, 6 were clustered together with $2 \mathrm{SCH}$ mice. Of the 9 $\mathrm{SCH}$ mice, 7 were clustered together with only $1 \mathrm{CON}$ mouse. Next, a hierarchical cluster was constructed using the expression values of the 36 identified proteins (Fig. 4B). The two groups exhibited marked separation, indicating that the liver proteins expressed in $\mathrm{SCH}$ mice were distinct from those in CON mice.
To investigate the correlation between altered miRNAs and proteins in the livers of SCH mice, the present study established a module between miRNAs and proteins using TargetScan and miRanda for prediction (Table II). The regulatory module contained 3 miRNAs, 4 proteins and 4 miRNA-protein connections. It was observed that miR-34a-5p targets thioredoxin (Txn), miR-130a-3p targets elongation factor 1-beta (Eef1b) and prosaposin (Psap), while miR-24-5p targets selenium-binding protein 2 (Selenbp2).

\section{Discussion}

In previous clinical studies, a positive association has been reported between TSH and serum TG levels (33). TG synthesis could be induced by TSH through GPAT3 in adipocytes (34), while TSH has been demonstrated to promote hepatic TG accumulation by increasing SREBP-1c activity (12). In order to further investigate the effect of TSH on hepatic lipid metabolism, a noninvasive method of MMI administration in the drinking water to successfully establish SCH mouse 
Table II. Targets of differentially expressed miRNAs in subclinical hypothyroidism mice.

\begin{tabular}{|c|c|c|}
\hline miRNA & $\begin{array}{l}\text { Putative } \\
\text { target gene }\end{array}$ & Definition \\
\hline mmu-miR-24-3p & Selenbp2 & $\begin{array}{l}\text { Selenium-binding } \\
\text { protein } 2\end{array}$ \\
\hline mmu-miR-34a-5p & Txn & Thioredoxin \\
\hline \multirow[t]{2}{*}{ mmu-miR-130a-3p } & Eef1b & Elongation factor $1-\beta$ \\
\hline & Psap & Prosaposin \\
\hline
\end{tabular}

miRNA/miR, microRNA.

model (4). miRNAs have been studied in a variety of liver diseases, including viral hepatitis, cirrhosis, hepatoma and NAFLD (7,35). However, their application is challenging, as miRNAs have different and intersecting target genes (5). Previous research has revealed that modules containing genes and targeting regulators could be used as diagnostic and therapeutic tools (36). Therefore, it was hypothesized that miRNA and proteome profiles could be integrated to form an miRNA-protein regulatory module, which may be associated with hepatic lipid metabolism disorders in $\mathrm{SCH}$ and thereby be used to explore potential therapeutic targets.

In the present study, a total of 17 hepatic miRNAs that have been confirmed as crucial gene regulators of hepatic lipid metabolism were selected to explore their profiles in $\mathrm{SCH}$, among them, miR-10b regulates hepatocyte steatosis by targeting peroxisome proliferator-activated receptor- $\alpha$ (15). In addition, miR-24 and miR-125b regulate hepatic lipid accumulation by targeting insulin-induced gene 1 and fatty acid synthase (FAS), respectively $(16,20)$. miR-29 and miR-486 are reported to regulate cholesterol metabolism by targeting hydroxy-3-methyl-glutaryl-CoA reductase and histone acetyltransferase-1, respectively $(17,28)$. Furthermore, miR-30b-5p and miR-30c-5p belong to the same family of miRNAs regulating fatty acid synthesis genes, targeting elongation of very long chain fatty acids protein 5 (ELOVL5) and fatty acid synthase (FAS), respectively $(18,19)$. miR-34a has been reported to participate in proinflammatory NAFLD (24), while miR-130a-3p directly targets transforming growth factor- $\beta$ receptors 1 and 2 , which may contribute to hepatic fibrosis (21). Additionally, miR-148a regulates cholesterol and TG homeostasis by controlling multiple metabolic regulatory circuits (30). miR-155 and miR-467b modulate hepatic steatosis by targeting liver $\mathrm{X}$ receptor $\alpha$ and hepatic lipoprotein lipase $(22,23)$. It has also been demonstrated that miR-199a-5p and miR-370 were implicated in fatty acid $\beta$-oxidation in mitochondria $(25,27)$. In addition, by simultaneously regulating insulin signaling and adipogenesis, miR-206 reduced lipid and glucose production in the liver of obese mice (29). In liver, miR-291b-3p promotes lipogenesis by suppressing AMPK $\alpha 1$ expression and activity (31). Hepatic miR-210 is elevated in cholestatic mice and PBC patients, promoting bile acids-induced liver injury by targeting mixed-lineage leukemia-4 (MLL4) (32). In the present study, it was observed that 8 (miR-10b-5p, miR-24-3p, miR-29a-3p, miR-30b-5p, miR-34a-5p, miR-125b-5p, miR-130a-5p and miR-199a-5p) out of these 17 miRNAs associated with hepatic lipid metabolic disorders were downregulated in the livers of $\mathrm{SCH}$ mice, suggesting that these miRNAs may be involved in hepatic lipid metabolic disorders in $\mathrm{SCH}$.

The present study subsequently conducted iTR AQ labeling analysis and identified 36 proteins with altered expression levels. Targeting predictions of miRNAs by TargetScan and miRanda were used to identify the potential targets (37). Of the significantly altered proteins, four were found to be potential targets of 3 differentially expressed miRNAs, namely the Txn, Eef1b, Psap and Selenbp2 proteins. It has been reported that the the Txn protein, has notable properties as a crucial defense against oxidative stress (38). Txn forms a system with thioredoxin reductase and nicotinamide adenine dinucleotide phosphate, and further eliminates reactive oxygen species, the excessive production of which leads to oxidative stress contributing to cardiac dysfunction and insulin resistance in NAFLD (39). TSH is known to directly produce oxidative stress, and oxidative damage to lipid peroxidation has been reported in $\mathrm{SCH}$ patients (40). The present study demonstrated marked alterations in miR-34a-5p and Txn levels in SCH mice, which may be involved in the development of hepatic lipid metabolism disorders in these mice via oxidative stress.

Eeflb, an enzyme that may be localized in the endoplasmic reticulum, has been reported to promote protein synthesis, and protect Leishmania major from chemical and oxidative stress (41). Furthermore, it is known that oxidative damage leads to lipid peroxidation, which is a key component of $\mathrm{SCH}$ and NAFLD (42). Thus, Eef1b, as the target of miR-130a-3p, may be involved in hepatic lipid metabolism disorders in $\mathrm{SCH}$ mice through lipid peroxidation.

Several minerals and trace elements are essential for normal thyroid hormone metabolism, such as iodine, iron and selenium, and hypothyroidism can easily arise in regions of severe iodine and selenium deficiency (43). However, to the best of our knowledge, the roles of Selenbp2 and Psap in hepatic lipid metabolism in SCH have not yet been studied; thus, the underlying mechanism requires further exploration.

In conclusion, the present study identified a miRNA-protein regulatory module, which included 3 miRNAs and 4 proteins, that may be associated with hepatic lipid metabolism by integrating miRNA and proteome profiles in $\mathrm{SCH}$ mice. To the best of our knowledge, no previous studies have investigated miRNAs that are involved in hepatic lipid metabolism in $\mathrm{SCH}$, and the present study may thus provide potential therapeutic targets and significant evidence for researchers to better understand the underlying pathogenesis of hepatic lipid metabolism in SCH. However, further investigation will be necessary in the future to verify these findings.

\section{Acknowledgements}

The abstract of this study was presented at the Endocrine Society Annual Meeting ENDO 2019, 22-23rd March 2019, New Orleans, LA, USA and was published as abstract number SAT-047. 


\section{Funding}

This study was supported by grants from the National Key Research and Development Program of China (no. 2017YFC1309800), the National Basic Research Program (no. 2012CB524900), the National Natural Science Foundation (nos. 81230018, 81471006, 81430020 and 81500595) and the Taishan Scholar's Special Expert Plan.

\section{Availability of data and materials}

The datasets used and/or analyzed during the current study are available from the corresponding author on reasonable request.

\section{Authors' contributions}

LZ performed the animal experiments and wrote the draft of the manuscript. KW, TB and LZ performed the molecular biology experiments. LG helped to develop the manuscript and interpreted the results. XZ and WC designed the study and performed data analysis. All authors approved the final manuscript for publication.

\section{Ethics approval and consent to participate}

All animal experiment procedures were approved by the Shandong Provincial Hospital Animal Care and Use Committee (approval no. 2015-003) and were in compliance with the Guide for the Care and Use of Laboratory Animals.

\section{Patient consent for publication}

Not applicable.

\section{Competing interests}

The authors declare that they have no competing interests.

\section{References}

1. Surks MI, Ortiz E, Daniels GH, Sawin CT, Col NF, Cobin RH, Franklyn JA, Hershman JM, Burman KD, Denke MA, et al: Subclinical thyroid disease: Scientific review and guidelines for diagnosis and management. JAMA 291: 228-238, 2004.

2. Quinn TJ, Gussekloo J, Kearney P, Rodondi N and Stott DJ: Subclinical thyroid disorders. Lancet 380: 335-337, 2012.

3. Xu L, Ma H, Miao M and Li Y: Impact of subclinical hypothyroidism on the development of non-alcoholic fatty liver disease: A prospective case-control study. J Hepatol 57: 1153-1154, 2012.

4. Zhou L, Ding S, Li Y, Wang L, Chen W, Bo T, Wu K, Li C, Liu X, Zhao J, et al: Endoplasmic reticulum stress may play a pivotal role in lipid metabolic disorders in a novel mouse model of subclinical hypothyroidism. Sci Rep 6: 31381, 2016.

5. Rottiers V and Näär AM: MicroRNAs in metabolism and metabolic disorders. Nat Rev Mol Cell Biol 13: 239-250, 2012.

6. Filipowicz W, Bhattacharyya SN and Sonenberg N: Mechanisms of post-transcriptional regulation by microRNAs: Are the answers in sight? Nat Rev Genet 9: 102-114, 2008.

7. Baffy G: MicroRNAs in nonalcoholic fatty liver disease. J Clin Med 4: 1977-1988, 2015.

8. Lu Y, Wang J, Guo X, Yan S and Dai J: Perfluorooctanoic acid affects endocytosis involving clathrin light chain $\mathrm{A}$ and microRNA-133b-3p in mouse testes. Toxicol Appl Pharmacol 318 41-48, 2017.
9. Chen W, Zhao W, Yang A, Xu A, Wang H, Cong M, Liu T, Wang P and You H: Integrated analysis of microRNA and gene expression profiles reveals a functional regulatory module associated with liver fibrosis. Gene 636: 87-95, 2017.

10. Li Y, Wang L, Zhou L, Song Y, Ma S, Yu C, Zhao J, Xu C and Gao L: Thyroid stimulating hormone increases hepatic gluconeogenesis via CRTC2. Mol Cell Endocrinol 446: 70-80, 2017.

11. Livak KJ and Schmittgen TD: Analysis of relative gene expression data using real-time quantitative PCR and the 2(-Delta Delta C(T)) method. Methods 25: 402-408, 2001.

12. Yan F, Wang Q, Lu M, Chen W, Song Y, Jing F, Guan Y, Wang L, Lin Y, Bo T, et al: Thyrotropin increases hepatic triglyceride content through upregulation of SREBP-1c activity. J Hepatol 61: 1358-1364, 2014

13. Calderon-Gonzalez KG, Valero Rustarazo ML, Labra-Barrios ML, Bazán-Méndez CI, Tavera-Tapia A, Herrera-Aguirre ME, Sánchez del Pino MM, Gallegos-Pérez JL, González-Márquez H, Hernández-Hernández JM, et al: Determination of the protein expression profiles of breast cancer cell lines by quantitative proteomics using iTRAQ labelling and tandem mass spectrometry. J Proteomics 124: 50-78, 2015.

14. Lewis BP, Burge CB and Bartel DP: Conserved seed pairing, often flanked by adenosines, indicates that thousands of human genes are microRNA targets. Cell 120: 15-20, 2005.

15. Zheng L, Lv GC, Sheng J and Yang YD: Effect of miRNA-10b in regulating cellular steatosis level by targeting PPAR-alpha expression, a novel mechanism for the pathogenesis of NAFLD. J Gastroenterol Hepatol 25: 156-163, 2010.

16. Ng R, Wu H, Xiao H, Chen X, Willenbring H, Steer CJ and Song G: Inhibition of microRNA-24 expression in liver prevents hepatic lipid accumulation and hyperlipidemia. Hepatology 60: 554-564, 2014.

17. Liu MX, Gao M, Li CZ, Yu CZ, Yan H, Peng C, Li Y, Li CG, Ma ZL, Zhao Y, et al: Dicer1/miR-29/HMGCR axis contributes to hepatic free cholesterol accumulation in mouse non-alcoholic steatohepatitis. Acta Pharmacol Sin 38: 660-671, 2017.

18. Zhang M, Li CC, Li F, Li H, Liu XJ, Loor JJ, Kang XT and Sun GR: Estrogen promotes hepatic synthesis of long-chain polyunsaturated fatty acids by regulating ELOVL5 at post-transcriptional level in laying hens. Int J Mol Sci 18: E1405, 2017.

19. Fan J, Li H, Nie X, Yin Z, Zhao Y, Chen C and Wen Wang D: MiR-30c-5p ameliorates hepatic steatosis in leptin receptor-deficient $(\mathrm{db} / \mathrm{db})$ mice via down-regulating FASN. Oncotarget 8 : 13450-13463, 2017.

20. Zhang ZC, Liu Y, Xiao LL, Li SF, Jiang JH, Zhao Y, Qian SW, Tang QQ and Li X: Upregulation of miR-125b by estrogen protects against non-alcoholic fatty liver in female mice. J Hepatol 63: 1466-1475, 2015.

21. Wang Y, Du J, Niu X, Fu N, Wang R, Zhang Y, Zhao S, Sun D and Nan Y: MiR-130a-3p attenuates activation and induces apoptosis of hepatic stellate cells in nonalcoholic fibrosing steatohepatitis by directly targeting TGFBR 1 and TGFBR2. Cell death Dis 8: e2792, 2017.

22. Ahn J, Lee H, Chung $\mathrm{CH}$ and Ha T: High fat diet induced downregulation of microRNA-467b increased lipoprotein lipase in hepatic steatosis. Biochem Biophys Res Commun 414: 664-669, 2011.

23. Miller AM, Gilchrist DS, Nijjar J, Araldi E, Ramirez CM, Lavery CA, Fernández-Hernando C, McInnes IB and Kurowska-Stolarska M: MiR-155 has a protective role in the development of non-alcoholic hepatosteatosis in mice. PLoS One 8: e72324, 2013.

24. Castro RE,Ferreira DM, Afonso MB, Borralho PM, Machado MV, Cortez-Pinto $\mathrm{H}$ and Rodrigues CM: miR-34a/SIRT1/p53 is suppressed by ursodeoxycholic acid in the rat liver and activated by disease severity in human non-alcoholic fatty liver disease. J Hepatol 58: 119-125, 2013.

25. Li B, Zhang Z, Zhang H, Quan K, Lu Y, Cai D and Ning G: Aberrant miR199a-5p/caveolin1/PPAR $\alpha$ axis in hepatic steatosis. J Mol Endocrinol 53: 393-403, 2014.

26. Zhang B, Wang R, Du J, Niu J, Zhang R, Xu S, Niu X, Zhang Q and Nan Y: Upregulated microRNA-199a-5p inhibits nuclear receptor corepressor 1 translation in mice with nonalcoholic steatohepatitis. Mol Med Rep 10: 3080-3086, 2014.

27. Iliopoulos D, Drosatos K, Hiyama Y, Goldberg IJ and Zannis VI: MicroRNA-370 controls the expression of microRNA-122 and Cptlalpha and affects lipid metabolism. J Lipid Res 51: $1513-1523,2010$ 
28. Liu D, Zhang M, Xie W, Lan G, Cheng HP, Gong D, Huang C, Lv YC, Yao F, Tan YL, et al: MiR-486 regulates cholesterol efflux by targeting HAT1. Biochem Biophys Res Commun 472: 418-424, 2016

29. Wu H, Zhang T, Pan F, Steer CJ, Li Z, Chen X and Song G: MicroRNA-206 prevents hepatosteatosis and hyperglycemia by facilitating insulin signaling and impairing lipogenesis. J Hepatol 66: 816-824, 2017.

30. Wagschal A, Najafi-Shoushtari SH, Wang L, Goedeke L, Sinha S, deLemos AS, Black JC, Ramírez CM, Li Y, Tewhey R, et al: Genome-wide identification of microRNAs regulating cholesterol and triglyceride homeostasis. Nat Med 21: 1290-1297, 2015.

31. Meng X, Guo J, Fang W, Dou L, Li M, Huang X, Zhou S, Man Y, Tang W, Yu L and Li J: Liver MicroRNA-291b-3p promotes hepatic lipogenesis through negative regulation of adenosine 5 '-monophosphate (AMP)-activated protein kinase $\alpha 1$. J Biol Chem 291: 10625-10634, 2016.

32. Kim YC, Jung H, Seok S, Zhang Y, Ma J, Li T, Kemper B and Kemper JK: MicroRNA-210 promotes bile acid-induced cholestatic liver injury by targeting mixed-lineage leukemia-4 methyltransferase in mice. Hepatology: Sep 24, 2019 (Epub ahead of print)

33. Wanjia X, Chenggang W, Aihong W, Xiaomei Y, Jiajun Z, Chunxiao Y, Jin X, Yinglong H and Ling G: A high normal TSH level is associated with an atherogenic lipid profile in euthyroid non-smokers with newly diagnosed asymptomatic coronary heart disease. Lipids Health Dis 11: 44, 2012.

34. Ma S, Jing F, Xu C, Zhou L, Song Y, Yu C, Jiang D, Gao L, Li Y, Guan Q and Zhao J: Thyrotropin and obesity: Increased adipose triglyceride content through glycerol-3-phosphate acyltransferase 3. Sci Rep 5: 7633, 2015.

35. Louten J, Beach M, Palermino K, Weeks M and Holenstein G: MicroRNAs expressed during viral infection: Biomarker potential and therapeutic considerations. Biomarker Insights 10 (Suppl 4): S25-S52, 2015
36. Barabasi AL, Gulbahce N and Loscalzo J: Network medicine: A network-based approach to human disease. Nat Rev Genet 12 56-68, 2011.

37. Peterson SM, Thompson JA, Ufkin ML, Sathyanarayana P, Liaw L and Congdon CB: Common features of microRNA target prediction tools. Front Genet 5: 23, 2014.

38. Lu J and Holmgren A: The thioredoxin antioxidant system. Free Radic Biol Med 66: 75-87, 2014.

39. Yang J, Hamid S, Cai J, Liu Q, Xu S and Zhang Z: Selenium deficiency-induced thioredoxin suppression and thioredoxin knock down disbalanced insulin responsiveness in chicken cardiomyocytes through PI3K/Akt pathway inhibition. Cell Signal 38: 192-200, 2017.

40. Haribabu A, Reddy VS, Pallavi CH, Bitla AR, Sachan A, Pullaiah P, Suresh V, Rao PV and Suchitra MM: Evaluation of protein oxidation and its association with lipid peroxidation and thyrotropin levels in overt and subclinical hypothyroidism. Endocrine 44: 152-157, 2013

41. Sasikumar AN, Perez WB and Kinzy TG: The many roles of the eukaryotic elongation factor 1 complex. Wiley Interdiscip Rev RNA 3: 543-555, 2012.

42. Seki S, Kitada T, Yamada T, Sakaguchi H, Nakatani K and Wakasa K: In situ detection of lipid peroxidation and oxidative DNA damage in non-alcoholic fatty liver diseases. J Hepatol 37: $56-62,2002$.

43. Zimmermann MB and Kohrle J: The impact of iron and selenium deficiencies on iodine and thyroid metabolism: Biochemistry and relevance to public health. Thyroid 12: 867-878, 2002. International (CC BY-NC-ND 4.0) License. 